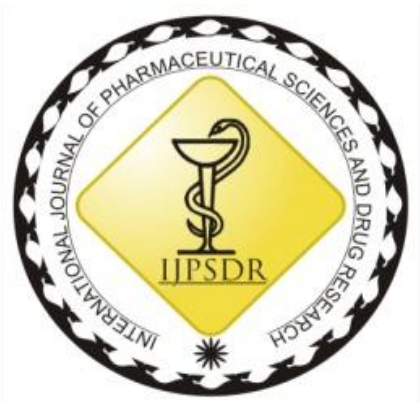

ISSN: 0975-248X

RESEARCH ARTICLE CODEN (USA): IJPSPP

$($ (c) $)$ EY-NC-SA

\title{
Design and in vivo Evaluation of Manidipine by Self- Nanoemulsifying Drug Delivery Systems
}

\author{
S. Srikanth Reddy ${ }^{1}$, G. Suresh ${ }^{2 *}$ \\ ${ }^{1}$ Career Point University, Kota-325003, Rajasthan, India \\ ${ }^{2}$ Aavyan labs, Pashamylaram, Hyderabad-502307, Telangana, India
}

Copyright (C) 2019 S. Srikanth Reddy et al. This is an open access article distributed under the terms of the Creative Commons AttributionNonCommercial-ShareAlike 4.0 International License which allows others to remix, tweak, and build upon the work non-commercially, as long as the author is credited and the new creations are licensed under the identical terms.

\begin{abstract}
The current research is aimed at developing liquid self-nanoemulsifying drug delivery system (liquid-SNEDDS) of Manidipine for enhanced solubility and oral bioavailability. The Manidipine SNEDDS are formulated with excipients comprising of Capmul MCM (oil phase), Transcutol P (surfactant) Lutrol L 300 as co-surfactant. The prepared fifteen formulations of Manidipine SNEDDS analysed for emulsification time, percentage transmittance, particle size, in vitro drug release, and stability studies. In vivo pharmacokinetic studies of the optimized formulation were carried out in Wistar rats in comparison with control (pure drug). The morphology of Manidipine SNEDDS indicates spherical shape with uniform particle distribution. The percentage drug release from optimized formulation F14 is $98.24 \pm$ 5.14\%. The particle size F14 formulation was $22.4 \mathrm{~nm}$ and Z-Average $23.3 \mathrm{~nm}$. The PDI and zeta potential of Manidipine SNEDDS optimized formulation (F14) were 0.313 and-5.1mV respectively. From in vivo bioavailability data the optimized formulation exhibited a significantly greater $\mathrm{C}_{\max }$ and $\mathrm{T}_{\max }$ of the SNEDDS was found to be $3.42 \pm$ $0.46 \mathrm{ng} / \mathrm{ml} \& 2.00 \pm 0.05 \mathrm{~h}$ respectively. $\mathrm{AUC}_{0-\infty}$ infinity for formulation was significantly higher $(11.25 \pm 3.45$ ng.h/ml) than pure drug (7.45 $\pm 2.24 \mathrm{ng} . \mathrm{h} / \mathrm{ml})$. Hence a potential SNEDDS formulation of Manidipine developed with enhanced solubility and bioavailability.
\end{abstract}

Keywords: Manidipine, Hypertension, SNEDDS, Solubility studies, Pharmacokinetic studies.

DOI: 10.25004/IJPSDR.2019.110609

Int. J. Pharm. Sci. Drug Res. 2019; 11(6): 337-342

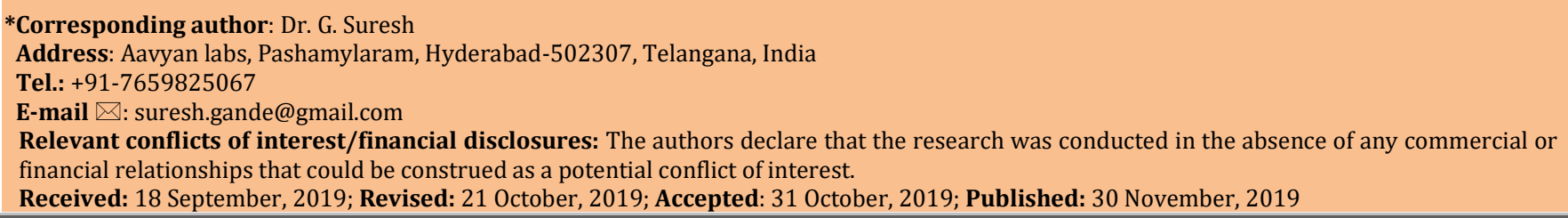

\section{INTRODUCTION}

The Class II to class IV drugs of Biopharmaceutical Classification System (BCS) suffering with poor water solubility lead to lower intestinal absorption, lower bio availability. Solubilising poor water soluble drugs is a major challenge in pharmaceutical research. Lipid based drug formulations increase the relative solubility of drugs in GI track by enhancing absorption. Self- emulsifying drug delivery systems (SNEDDS) lipid based formulations are most promising technology in drug delivery. [1-3] SNEDDS are defined as pre concentre containing a mixture of drug, surfactants, oil and co-surfactant. The smaller size of SNEDDS improves drug dissolution by increasing area for drug release, absorption and by promoting lymphatic transport of the drug. SNEDDS formulation is used for 
increasing the solubility, oral bioavailability and permeability of drug. It also protects the drug from hostile environment in GI track and is used for selective GI targeting drug delivery. [4-8] They exhibit particle size ranging from nano meters to few microns. Based on particle size they are further classified into SMEDDS and SNEDDS. SMEDDS form micro emulsions consisting of oil droplets size ranging 100 and $200 \mathrm{~nm}$ sixe. SNEDDS contain the droplets whose size is less than $100 \mathrm{~nm}$.

Manidipine is used as an antihypertensive that binds to voltage dependent calcium channels and dissociates them, thus blocking extracellular calcium from entering into cell. [9-10] This produces vasodilation hence decreasing the blood pressure. The objective of present research is to design and evaluate the liquid-SNEDDS of Manidipine. The ability of SNEDDS to improve dissolution rate, solubility and bioavailability is evaluated.

Table 1: Formulation trials of liquid Manidipine SNEDDS

\begin{tabular}{ccccccc}
\hline $\begin{array}{c}\text { S }_{\text {mix }} \\
\text { (Surfactant: } \\
\text { Cosurfactant) }\end{array}$ & Oil:S & Formulation & $\begin{array}{c}\text { Manidipine } \\
\text { (mg) }\end{array}$ & $\begin{array}{c}\text { Oil } \\
\text { Copmul } \\
\text { MCM)(ml) }\end{array}$ & $\begin{array}{c}\text { Smix } \\
\text { (Transcutol } \\
\text { P: Lutrol L } \\
\mathbf{3 0 0}) \\
(\mathbf{m l})\end{array}$ & Water \\
\hline & $1: 9$ & F1 & 10 & 0.15 & 1.35 & 1.1 \\
& $2: 8$ & F2 & 10 & 0.3 & 1.2 & 1.25 \\
1.1 & $3: 7$ & F3 & 10 & 0.45 & 1.05 & 1.4 \\
& $4: 6$ & F4 & 10 & 0.6 & 0.9 & 1.55 \\
& $5: 5$ & F5 & 10 & 0.75 & 0.75 & 1.7 \\
& $7: 3$ & F6 & 10 & 0.15 & 0.45 & 3.6 \\
& $8: 2$ & F7 & 10 & 1.2 & 0.3 & 3.82 \\
$2: 1$ & $9: 1$ & F8 & 10 & 1.35 & 0.15 & 3.95 \\
& $1: 9$ & F9 & 10 & 0.15 & 1.35 & 2.75 \\
& $2: 8$ & F10 & 10 & 0.3 & 1.2 & 2.89 \\
& $5: 5$ & F11 & 10 & 0.75 & 0.75 & 4.61 \\
& $6: 4$ & F12 & 10 & 0.9 & 0.6 & 4.72 \\
$3: 1$ & $7: 3$ & F13 & 10 & 1.05 & 0.45 & 4.86 \\
& $8: 2$ & F14 & 10 & 1.2 & 0.3 & 4.91 \\
& $9: 1$ & F15 & 10 & 1.35 & 0.15 & 5.2 \\
\hline
\end{tabular}

\section{MATREIALS AND METHODS}

\section{Materials}

Manidipine is gifted by Aurobindo Pharma limited, Hyderabad. Caproyl P GMC and Acrysol K-150 Oleic acid, Lauroglycol, Transcutol HP are procured from Gattifosse, Mumbai. Labrosol, Tween 20, Acconon, Lutrol L 300, Capmul CMC, Labrosol, Acconon and Lutrol L 300 generous samples from BASF, Mumbai.

\section{Solubility data}

The solubility of Manidipine in various oils, cosurfactants and surfactants were examined by adding Manidipine (approximately $10 \mathrm{mg}$ ) to $2 \mathrm{ml}$ each. The samples were further centrifuged $8,000 \mathrm{rpm}$ for $20-30$ min at $4^{\circ} \mathrm{C}$. The supernatant removed and the drug concentration determined by UV at $228 \mathrm{~nm}$. [11]

\section{Construction of ternary phase diagrams}

The phase diagram constructed base on the solubility of drug in various excipients. Various combinations of oil, surfactant and co-surfactant were considered for construction of the same. Various weight ratios of oil and $S_{\text {mix }}$ ranging from 1:9 to 9:1 $(1: 9,2: 8,3: 7,4: 6,5: 5,6: 4$, $7: 3,8: 2,9: 1)$ were taking glass vials. The exact ratio excipients in the SNEDDS formulation was analysed followed by construction of Pseudo ternary plots using Chemix software. [12-13]

Measurement of percentage Transmittance

The Manidipine SNEDDS reconstituted with distilled water. The resulting emulsion was visually examined for any turbidity. The percentage transmittance measured using UV spectrophotometer at $228 \mathrm{~nm}$. [14]

\section{Emulsification Time}

By applying virtual test method, about $0.2 \mathrm{ml}$ of mixture was diluted with $300 \mathrm{ml}$ of water at a temperature of $37^{\circ} \mathrm{C}$ using a magnetic stirrer. The tendency of emulsion formation observed. [15]

Development of Manidipine SNEDDS formulations

The Manidipine SNEDDS formulations prepared using Capmul MCM was used as oil phase and Transcutol P and Lutrol L 300 used as surfactant and co-surfactant (Table 1$)$. Manidipine $(10 \mathrm{mg})$ was added to oil into glass vial and heated to $40^{\circ} \mathrm{C}$. The oily mixture mixed thoroughly with surfactant and co-surfactant. The mixture sonicated for $15 \mathrm{~min}$.

Thermodynamic stability studies and drug content

The stable samples centrifuged at $3000 \mathrm{rpm}$ for $300 \mathrm{sec}$ and examined for any phase separation. The concentration of drug in $0.2 \mathrm{ml}$ of formulation was determined by UV spectrophotometric method. [16]

In vitro dissolution studies of Manidipine SNEDDS formulations

$10 \mathrm{mg}$ of liquid SNEDDS Manidipine formulation was filled into gelatin capsules and dissolution studies were undertaken, samples were withdrawn were analysed at $228 \mathrm{~nm}$ by UV. [4, 17]

Characterization of Manidipine SEDDS Formulation Droplet size determination

The droplet size of Manidipine SNEDDS formulations were determined using Malvern Instrument Photon correlation spectroscopy (UK) that measures the size range of 10 to $5000 \mathrm{~nm}$. [18]

\section{Zeta potential Determination}

The zeta potential of the diluted Manidipine SNEDDS formulation was determined by Zetasizer. [19]

Stability studies

The Manidipine SNEDDS formulations pilled in gelatin capsules. Stability studies conducted at $25^{\circ} \mathrm{C}$ temperature $/ 60 \% \mathrm{RH}$ and $40^{\circ} \mathrm{C}$ temperature $/ 75 \% \mathrm{RH}$ in Thermolab stability chambers (Mumbai, India). [20]

Pharmacokinetic study of Manidipine SNEDDS Animals

Healthy Wistar rats were (Weighing 150-180 g) selected for this study, all the animals were healthy during the period of the experiment. All efforts were made to maintain the animals under controlled environmental conditions (Temperature $25^{\circ} \mathrm{C}$, Relative Humidity $45 \%$ and $12 \mathrm{~h}$ alternate light and dark cycle) with $100 \%$ fresh air exchange in animal rooms, uninterrupted power and water supply. Rats were fed with standard diet and water ad libitum. The protocol was approved by institutional ethical committee with no 1292/ac/09/CPCSEA/24/A. 


\section{Study Design}

The rats were categorised into two groups randomly and fasted for about 24 hours prior to the experiments. After 4 hours of dosing, foods were reoffered. Group 1 was administered with pure Manidipine (as such) methanol suspension and group 2 was orally administered with Manidipine SNEDDS diluted methanol $(0.5 \%)$ a dose of $10 \mathrm{mg} / \mathrm{kg}$. Then, $500 \mu \mathrm{L}$ blood samples were collected from the femoral artery at certain times $0,0.50,1,1.50,2,2.50,3,4,5,6,8,12,16$, 20, $24 \mathrm{~h}$ post dose and transferred into Eppendorf tubes containing heparin. Plasma was separated by centrifugation of the blood at $5000 \mathrm{rpm}$ in cooling centrifuge for $5 \mathrm{~min}$ to 10 minutes and stored frozen at $-20^{\circ} \mathrm{C}$ until analysis. [21]

\section{HPLC determination of Manidipine in rat plasma}

Manidipine and internal standard (felodipine) were extracted with n-hexane. The HPLC separation carried out over a Hypersil ODS2 column with a mobile phase comprising of methanol-5 mM ammonium acetate solution containing $0.1 \% \mathrm{CH}_{3} \mathrm{COOH}(85: 15, \mathrm{v} / \mathrm{v})$. The flow rate maintained at $1 \mathrm{ml} / \mathrm{min}$ with a detection wavelength of $304 \mathrm{~nm}$. The retention times about 5.8 min for Manidipine and $5.6 \mathrm{~min}$ for IS. [22]

\section{Pharmacokinetic analysis}

The pharmacokinetic parameters employed to evaluate were maximum plasma concentration $\left(\mathrm{C}_{\max }\right)$, time to attain $C_{\max }$ i.e., $T_{\max }$ and $t_{1 / 2}$ values, area under plasma concentration-time curve from zero to the final sampling time $\left(A U C_{0-t}\right)$, area under plasma concentration-time curve from zero to infinity $\left(A U C_{0}\right.$ $\infty)$. $A U C_{0-\infty}$ was calculated using the formula

$$
\mathrm{AUC}_{0-\infty}=\mathrm{AUC}_{0-\mathrm{t}}+\mathrm{C}_{\mathrm{t}} / \mathrm{K}_{\mathrm{E}}
$$

Table 2: Pharmacokinetic Parameters

\begin{tabular}{ccc}
\hline Parameters & $\begin{array}{c}\text { Manidipine Pure } \\
\text { drug }\end{array}$ & $\begin{array}{c}\text { Manidipine } \\
\text { SNEDDS }\end{array}$ \\
\hline $\mathrm{C}_{\max }(\mathrm{ng} / \mathrm{ml})$ & $2.19 \pm 0.32$ & $3.42 \pm 0.46$ \\
$\mathrm{AUC}_{\text {0-t }}(\mathrm{ng} . \mathrm{h} / \mathrm{ml})$ & $4.75 \pm 1.98$ & $7.82 \pm 2.74$ \\
$\mathrm{AUC}_{0 \text {-inf }}(\mathrm{ng} . \mathrm{h} / \mathrm{ml})$ & $7.45 \pm 2.24$ & $11.25 \pm 3.45$ \\
$\mathrm{~T}_{\max }(\mathrm{h})$ & $2.00 \pm 0.05$ & $1.00 \pm 0.04$ \\
$\mathrm{t}_{1 / 2}(\mathrm{~h})$ & $4.02 \pm 0.01$ & $2.52 \pm 0.04$ \\
\hline
\end{tabular}

\section{RESULTS}

\section{Solubility of Manidipine}

The solubility of Manidipine tested in oils phases (Acrysol K-150, Oleic acid, Capryol PGMC, Capmul MCM and Labrafil), surfactants (Kolliphor ELP, Labrasol, Cremophor EL, Lauroglycol, Transcutol HP and Tween 20) and co-surfactants Propylene glycol, PEG 400, Acconon, Lutrol L300 and Span 20. The maximum solubility was observed in Capmul MCM $61.84 \mathrm{mg} / \mathrm{ml}, 41.22 \mathrm{mg} / \mathrm{ml}$ of Transcutol HP and 133.24 $\mathrm{mg} / \mathrm{ml}$ of Lutrol L300 (Figure 1-3).

Pseudo ternary phase diagram

From the solubility data Capmul MCM was chosen as oil, Transcutol P as surfactant and Lutrol L300 as cosurfactant for SNEDDS formulation. A ternary phase diagram plotted indicating that increase in concentration of surfactant and co-surfactant with oil further increases the self-emulsifying region (Figure 4$6)$.

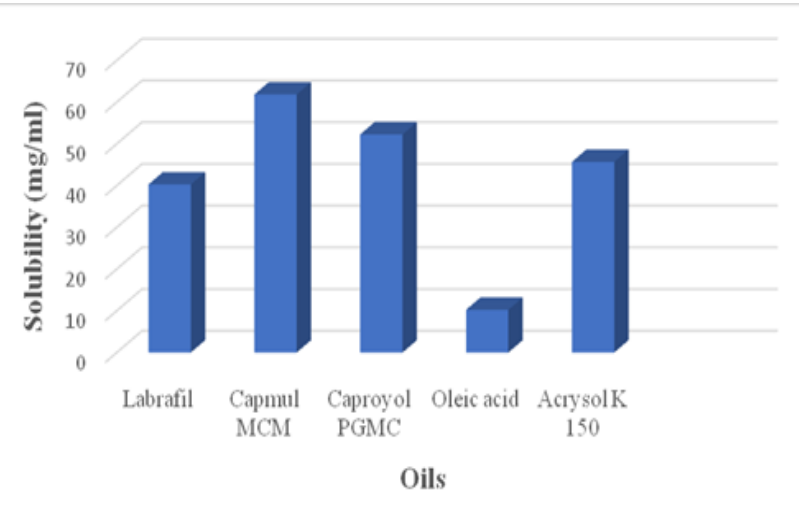

Fig. 1: Solubility of Manidipine in oils

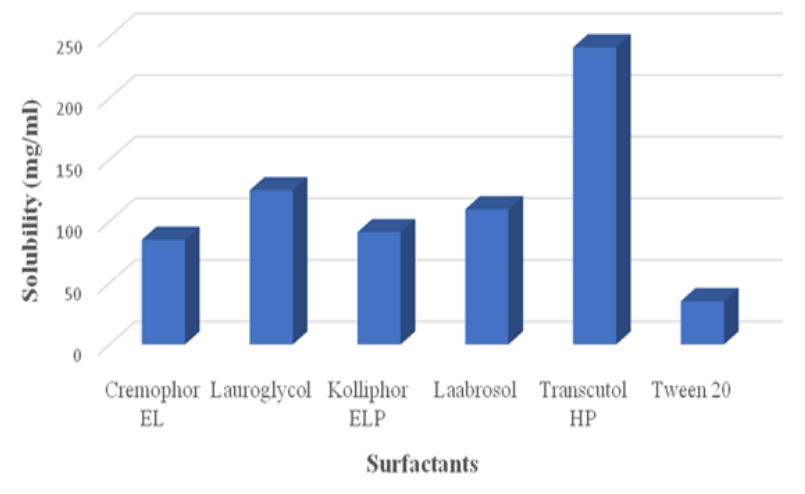

Fig. 2: Solubility of Manidipine in Surfactants

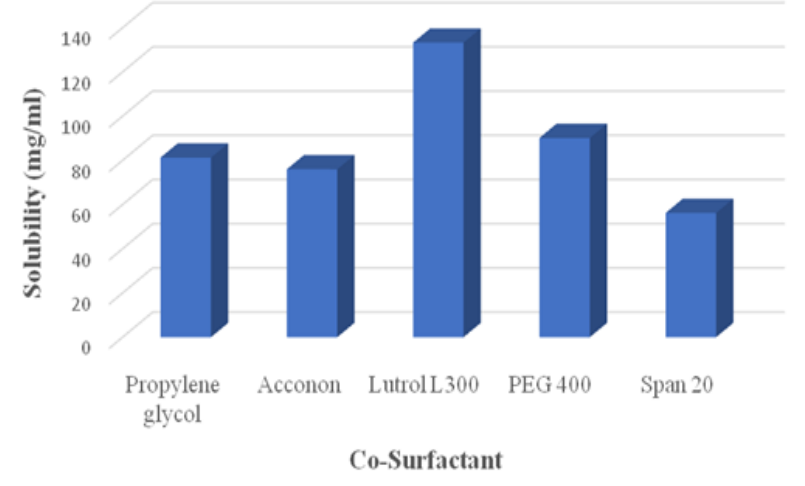

Fig. 3: Solubility of Manidipine in Co-surfactants

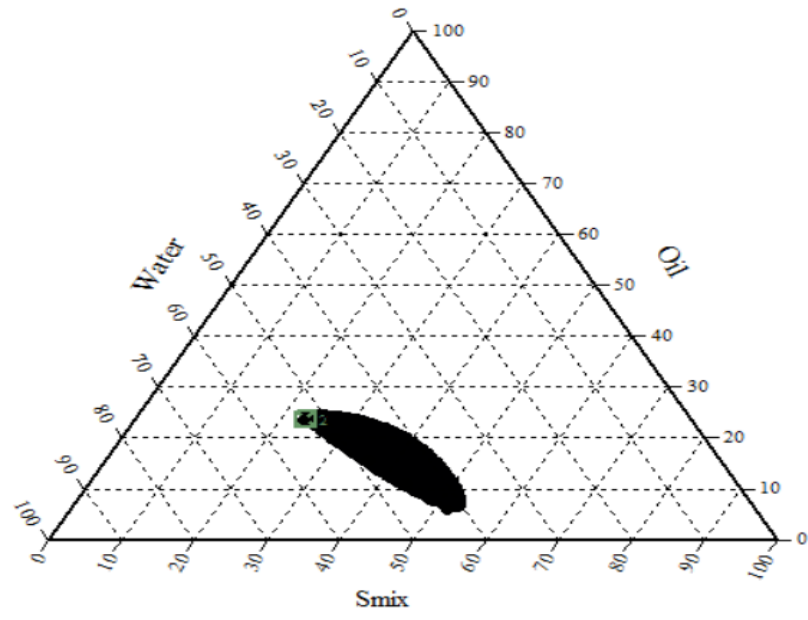

Fig. 4: Ternary phase diagram of Capmul MCM, Transcutol P and Lutrol L300 for 1:1 ratio of $S_{\text {mix }}$. 


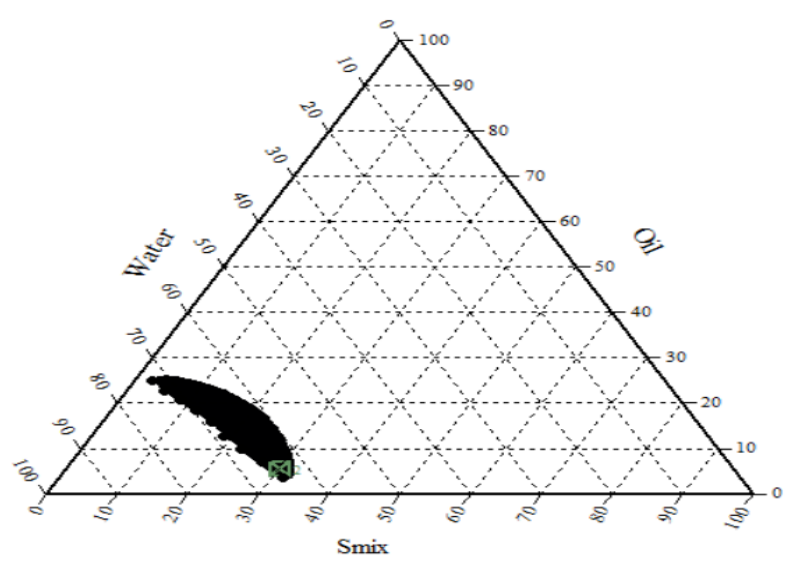

Fig. 5: Ternary phase diagram of Capmul MCM, Transcutol $P$ and Lutrol L300 for 2:1 ratio of $S_{\text {mix }}$.

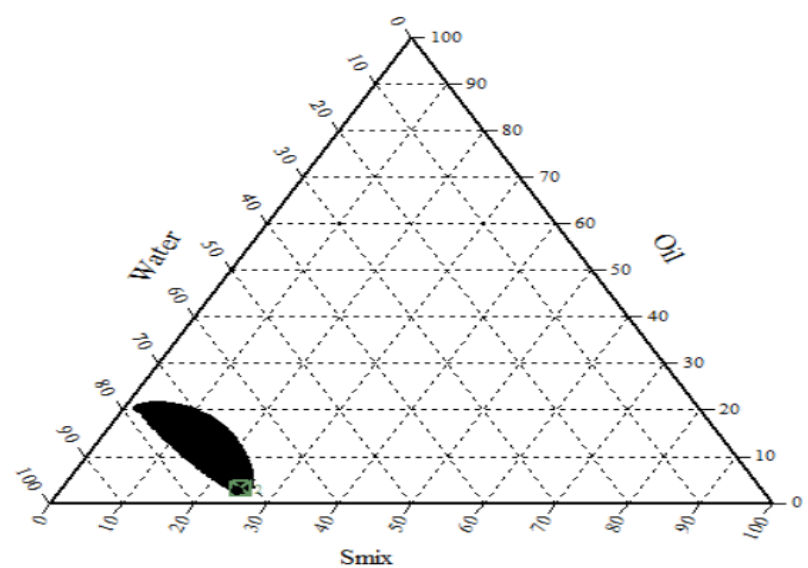

Fig. 6: Ternary phase diagram of Capmul MCM, Transcutol P and Lutrol L300 for 3:1 ratio of $S_{\text {mix }}$

Thermodynamic stability studies of Manidipine SNEDDS

The stability studies conducted indicated no significant phase separation or effect of temperature variation on physical appearance of the formulations. No significant change observed visually even after centrifugation freeze-thaw cycles.

$\%$ transmittance measurement and Drug content of Manidipine SEDDS

The transmittance $(\% \mathrm{~T})$ measures the clarity and transparency of emulsions. Formulation F14 exhibited $\%$ transmittance value $>99 \%$. The drug content in formulated Manidipine SNEDDS is within the range of $91.19-98.96 \%$. A maximum of $98.96 \%$ drug was observed in the formulation F14.

In-vitro dissolution studies of Manidipine SNEDDS The drug dissolution studies indicate that the drug release from F14 is higher than that of other fourteen formulations and the pure drug (Figure 7-9).

Characterization of Manidipine SEDDS

Particle size determination of Manidipine SNEDDS The droplet size and polydispersity values of Manidipine are analysed. The particle size of the optimized Manidipine SNEDDS formulation (F14) was found to be $22.4 \mathrm{~nm}$ and Z-Average $23.3 \mathrm{~nm}$. The results demonstrate that all the particles were in the nanometre range. The Polydispersity index of
Manidipine SNEDDS optimized formulation (F14) was 0.313. PDI determines the uniformity of particle diameter and hence it is useful to know the size distribution of nanoemulsion, which enhances good particle size distribution (Figure 10).

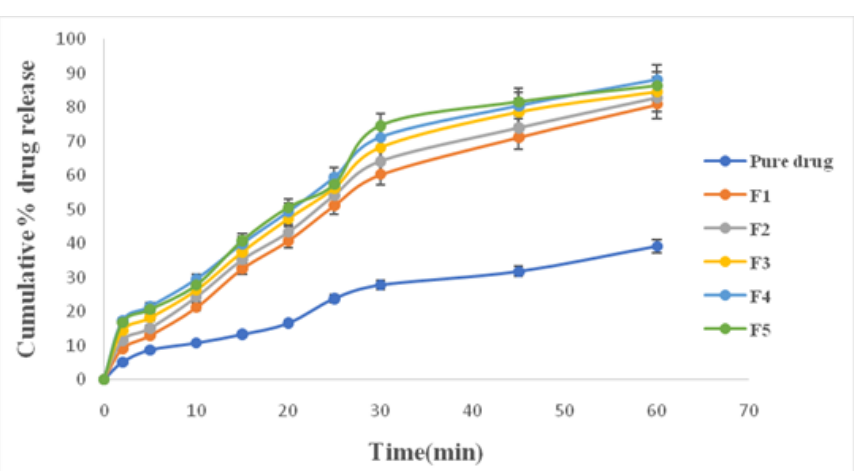

Fig. 7: Dissolution profiles of Manidipine pure drug and formulation F1-F5

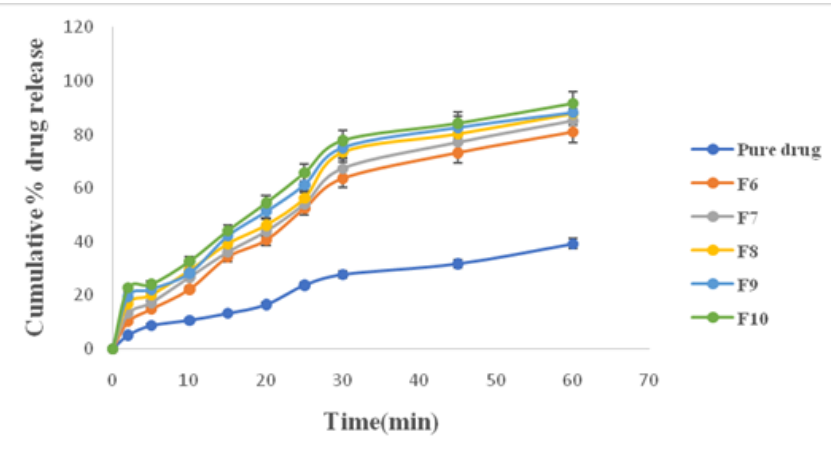

Fig. 8: Dissolution profiles of Manidipine pure drug and formulation F6- F10

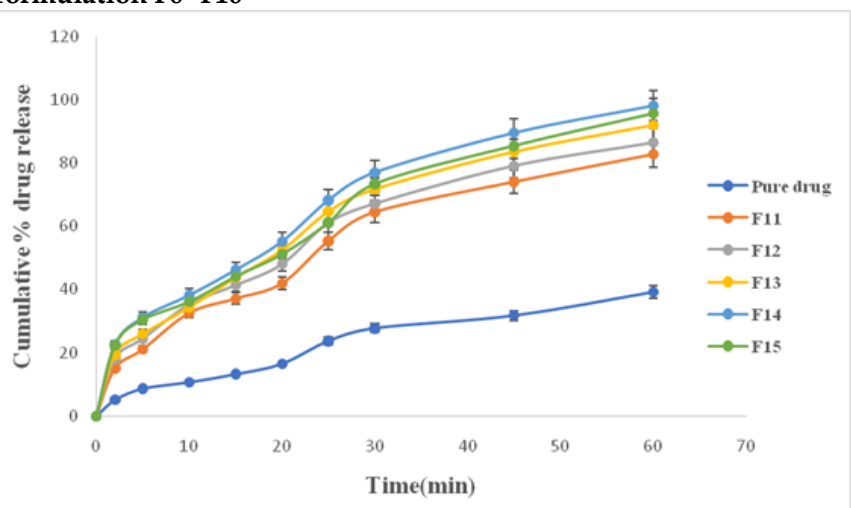

Fig. 9: Dissolution profiles of Manidipine pure drug and formulation F11 - F15)

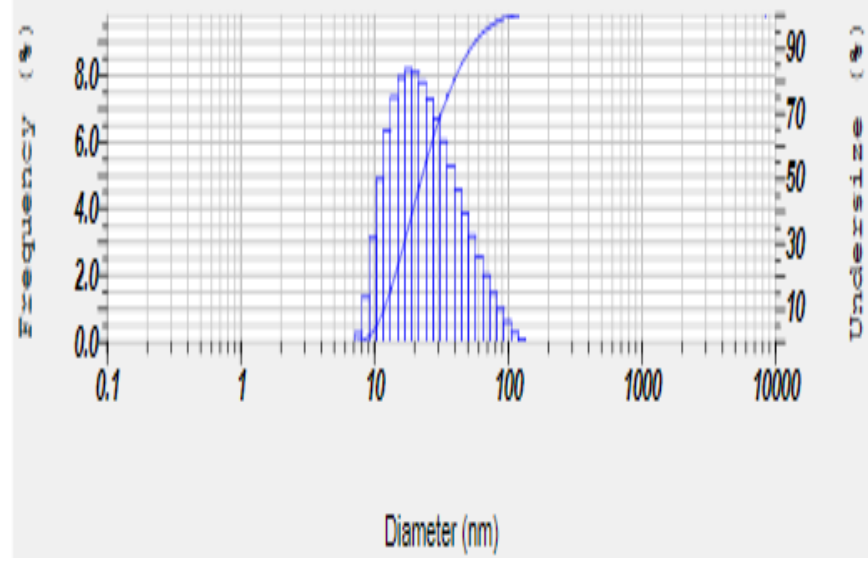

Fig. 10: Particle size analysis of Manidipine SNEDDS formulation F14 


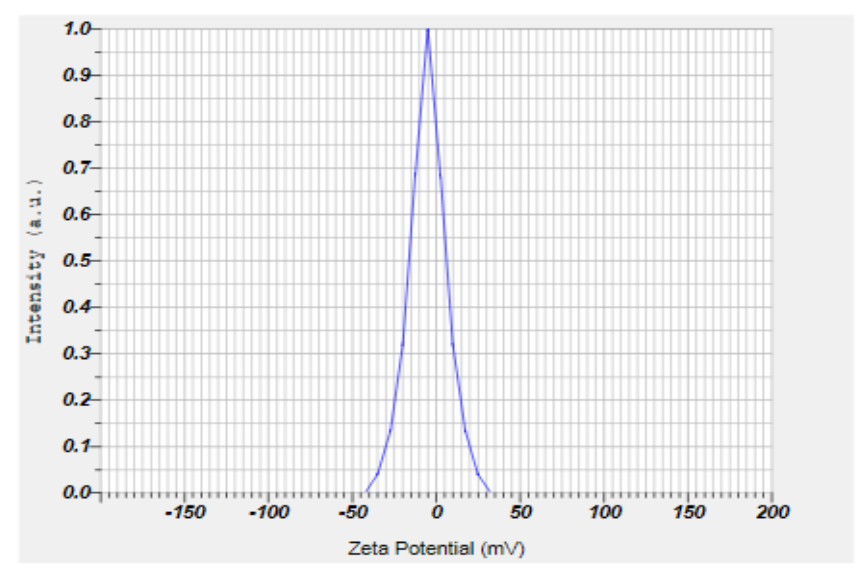

Fig. 11: Zeta potential of the Manidipine SNEDDS optimized formulation F14

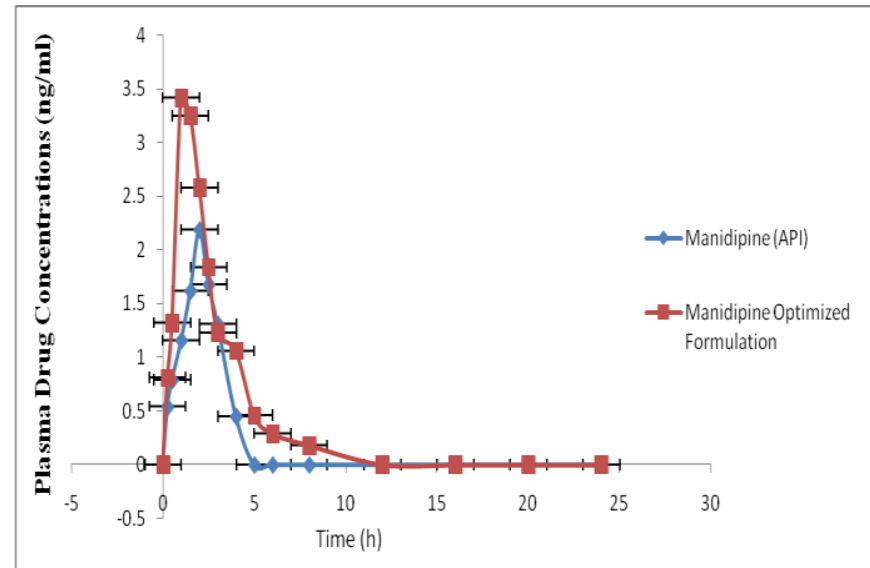

Fig. 12: Plasma concentration of Manidipine SNEDDS and pure drug

\section{Stability studies}

The stability of optimized Manidipine SNEDDS formulation studied for six months. The formulation was packed in hard gelatin capsules for the stability studies. The studies indicated no significant variation in drug release and the drug content, physical and emulsifying properties.

Zeta potential determination of Manidipine SNEDDS The zeta potential of the optimized SNEDDS formulation was $-5.1 \mathrm{mV}$ that is indicative of particle stability (Figure 11).

Pharmacokinetic parameters comparison for pure drug suspension and SNEDDS

Figure 12 shows the plasma concentration vs time curve in animals after single oral dose of Manidipine SNEDDS formulation in comparison with Manidipine pure suspension. At all the indicated time points, the Manidipine plasma concentration in animals treated with SNEDDS was found significantly greater than those treated with pure drug. Pharmacokinetic parameters of Manidipine in Wistar rats are shown in Table 2. $C_{\max }$ of the SNEDDS $3.42 \pm 0.46 \mathrm{ng} / \mathrm{ml}$ was significant $(p<0.05)$ when compared to the pure drug $2.19 \pm 0.32 \mathrm{ng} / \mathrm{ml}$. $T_{\max }$ of SNEDDS and pure drug were $2.00 \pm 0.05$ and $1.00 \pm 0.04 \mathrm{~h}$, respectively. $\mathrm{AUC}_{0-\infty}$ infinity for SNEDDS formulation was higher (11.25 \pm 3.45ng. $\mathrm{h} / \mathrm{ml}$ ) than the pure drug suspension formulation $7.45 \pm 2.24 \mathrm{ng} . \mathrm{h} / \mathrm{ml}$. Statistically, $\mathrm{AUC}_{0-\mathrm{t}}$ of
SNEDDS formulation was higher $(\mathrm{p}<0.05)$ than pure drug suspension. Higher amount of drug concentration in blood indicated better systemic absorption of Manidipine from SNEDDS formulation as compared to the pure drug suspension formulation.

The present research is aimed at formulation and characterization of Manidipine SNEDDS. The solubility studies indicated maximum solubility of drug in Capmul MCM (oil), Transcutol HP (Surfactant) and Lutrol L300 (co surfactant). A tertiary phase diagram plotted in accordance with solubility studies indicate enhancement of self-emulsifying region was with increase in concentration of surfactant and co surfactant with oil. Base on the results fifteen Manidipine liquid SNEDDS formulations F1-F15 were prepared and analysed. The thermodynamic stability studies of all formulation indicate no change in visual description of samples. The \% transmittance study indicates Manidipine liquid SNEDDS formulation F14 has a value $>99 \%$ indicating high clarity of emulsion. Among all F14 was chosen as optimized formulation of Manidipine based on the drug content and dissolution studies. The particle size of F14 is $22.4 \mathrm{~nm}$, PI 0.313 and Zeta potential $-5.1 \mathrm{mv}$. The SEM studies of optimized formulation F14 indicated spherical shape with uniform particle distribution. The formulation F14 subjected to stability studies for six months indicated no significant variation in drug content, drug release, emulsifying properties and appearance. From in vivo bioavailability studies the optimized formulation was exhibited a significantly greater $\mathrm{C}_{\max }$ than the pure drug suspension. $\mathrm{AUC}_{0-\infty}$ for SNEDDS formulation was higher than the pure drug suspension formulation. Statistically, $\mathrm{AUC}_{0-\mathrm{t}}$ of the SNEDDS formulation was significantly higher $(p<0.05)$ as compared to pure drug suspension formulation. Hence a potential liquid SNEDDS formulation of Manidipine developed with enhanced solubility, dissolution rate and bioavailability.

\section{REFERENCES}

1. Charman WN, Stella VJ. Transport of lipophilic molecules by the intestinal lymphatic system. Adv Drug Del Rev. 1991; 7:114.

2. O'Driscoll CM. Lipid-based formulations for intestinal lymphatic delivery. Eur J Pharm Sci. 2002; 15:405-415.

3. Porter CJ, Trevaskis NL, Charman WN. Lipids and lipidbased formulations: optimizing the oral delivery of lipophilic drugs. Nat Rev Drug Discov. 2007; 6:231-248.

4. Shafiq S, Shakeel F, Talegaonkar S, Ahmad FJ, Khar RK Development and bioavailability assessment of Ramipril nanoemulsion formulation. Eur J Pharm Biopharm. 2007; 66:227-243.

5. Attivi D, Ajana I, Astier A, Demoré B, Gibaud S. Development of microemulsion of mitotane for improvement of oral bioavailability. Drug Dev Ind Pharm. 2010; 36:421-427.

6. Dixit AR, Rajput SJ, Patel SG. Preparation and bioavailability assessment of SMEDDS containing valsartan. AAPS PharmSciTech. 2010; 11:314-321.

7. Nielsen FS, Petersen KB, Müllertz A. Bioavailability of probucol from lipid and surfactant based formulations in minipigs: influence of droplet size and dietary state. Eur J Pharm Biopharm. 2008; 69:553-562. 
8. Wu $\mathrm{X}, \mathrm{Xu}$ J, Huang $\mathrm{X}$, Wen C .Self-microemulsifying drug delivery system improves curcumin dissolution and bioavailability. Drug Dev Ind Pharm 2011; 37:15-23.

9. Cui J, Yu B, Zhao Y, Zhu W, Li H. Enhancement of oral absorption of curcumin by self-micro emulsifying drug delivery systems. Int J Pharm. 2009; 371:148-155.

10. McKeage K, Scott LJ. Manidipine a review of its use in the management of hypertension. Drugs 2004; 64(17):1923-40.

11. Cheer SM, McClellan K. Manidipine a review of its use in hypertension. Drugs 2001; 61(12):1777-99.

12. Chaudhari $\mathrm{P}$, Bhalerao A. Method validation for spectrophotometric estimation of cilnidipine. Int J Pharm PharmSci. 2012; 4(5):96-98.

13. Talegaonkar S, Mustafa G, Akhter S, Iqbal ZI. Design and Development of Oral Oil-in-Water Nanoemulsion Formulation Bearing Atorvastatin: In Vitro Assessment. J DispersSci Technol.2010; 31(5):690-701.

14. Setthacheewakul S, Mahattanadul S, Phadoongsombut N, Pichayakorn W, Wiwattanaptapee R. Development and Characterization of Solid Self-emulsifying Drug Delivery System of Cilnidipine. Eur J Pharm Biopharm. 2010; 76:475485.

15. Constantinides PP, Scalart JP, Lancaster C, Marcello J, Marks G, Ellens H, Smith PL. Formulation and Intestinal Absorption Enhancement Evaluation of Water-in-Oil Microemulsions Incorporating Medium-Chain Glycerides. Pharmaceutical Research 1994; 11(10):1385-1390.
16. Rajinikanth PS, Keat NW, Garg S. Self-nanoemulsifying drug delivery systems of valsartan: Preparation and in-vitro characterization. International Journal of Drug Delivery 2012; 4(2):153-163.

17. Costa P, Sousa Lobo JM. Modeling and comparison of dissolution profiles. Eur J Pharm Sci. 2001; 13:123-133.

18. Dixit P, Jain DK, Dumbwani J. Standardization of an ex vivo method for determination of intestinal permeability of drugs using everted rat intestine apparatus. J Pharmacol Toxicol Methods. 2012; 65:13-17.

19. Shaji J, Jadhav D. Newer Approaches to Self Emulsifying Drug Delivery System. Int J Pharm PharmSci 2010; 2:37-42.

20. Sonia A, Rishikesh G, Prajapati S. Self-Micro emulsifying Drug Delivery System. Asian J Pharm Clin Res. 2016; 9(2):3338.

21. Zhang J, Peng Q, Shi S, Zhang Q, Sun X, Gong T, Zhang Z. Preparation, characterization, and in vivo evaluation of a selfnanoemulsifying drug delivery system (sNeDDs) loaded with morin-phospholipid complex. Int J Nanotechnol Nanomed. 2011; 6:3405-14.

22. Jing J, Ren W, Chen $X$, He H, Zhou W, Zhu X, Sun Y, Wang G. Determination and pharmacokinetics of manidipine in human plasma by HPLC/ESIMS. Biomed Chromatogr. 2007; 21(8): 836-840. 